\title{
PASSIFICATION OF NONSQUARE LINEAR SYSTEMS
}

\author{
Alexander Fradkov \\ Institute for Problems of Mechanical Engineering, \\ Russian Academy of Sciences, \\ 61 Bolshoy ave V.O., St. Petersburg, 199178, RUSSIA \\ Fax : +7 (812) 321-4771, E-mail : alf@ipme.ru
}

Keywords: Passification, Linear systems, YakubovichKalman-Popov lemma.

\begin{abstract}
Necessary and sufficient conditions for feedback passivity (passifiablity) of nonsquare linear systems published in Russian and Western literarure are surveyed. New output $G$ passifiability conditions for nonsquare linear systems are given. The proofs are based on Yakubovich-Kalman-Popov (KalmanYakubovich) lemma.
\end{abstract}

\section{Introduction}

During last decade there was a continuing interest in passifiable or feedback passive systems - ones that can be made passive by means of state or output feedback $[1,2,3,4,5,6]$. Since passivity of a linear system is equivalent to positive realness of its transfer function, the linear passifiable (strictly passifiable) systems were also called "almost positive real" ("almost strictly positive real") [7, 8].

For output feedback case necessary and sufficient conditions of passifiability were suggested in [9, 10] (for SISO systems) and in $[11,12,13]$ for MIMO systems. Note that feedback structures in [11, 12] and in [13] are different (see also [14]). State feedback case was considered in $[15,1]$. In [16] it was shown that conditions of strict passifiability for state feedback and output feedback case are the same. In a number of works the problem of positive real synthesis for systems with feedthrough (relative degree zero case) was considered, see [17] and references therein. The obtained results have applications in robust control $[9,10,11,12]$, adaptive control $[8,20,13]$, stabilization of partially linear cascaded systems $[15,1,5]$.

In the Russian literature the problem of feedback design ensuring strict positive realness was studied still in the 1970s, see [18] for SIMO systems and [19] for MIMO systems. The results were systematically applied to adaptive control [21, 22, 23], to VSS design [24], to synchronization systems design $[25,26,27]$. The necessary and sufficient conditions of output strict feedback passivity in [9, 10, 11, 12, 13] are straightforward consequences of the corresponding results of $[18,19]$. Although the papers [18, 19] (translations from Russian) were published in the West and some results of $[18,19]$ were recalled in $[28,20,16]$, these results were somewhat overlooked in the Western literature.
In this note the main results of [19] giving necessary and sufficient output feedback passivity conditions for nonsquare linear systems are recalled. Also some new output passifiability conditions for nonsquare linear systems are given. Simple parametrization of a broad subclass of passifying feedbacks is proposed (see Corollary 2).

Despite common opinion that squaring down preserves all essential properties of the system, see [29], in some cases controller design without squaring down is preferable. Indeed, it provides designer with more flexibility and ease of interpreting the controller parameters. For adaptive control systems the non-squaring down designs lead to better parameter convergence: it is known that parameters converge faster and limit values are smaller [21, 23]. Therefore passification results given below can be useful for design purposes in various situations.

The problem is formulated in Section 2 containing also appropriate passivity definitions for nonsquare systems. The main results are presented in Section 3, while the proofs are given in the Section 4.

\section{Problem statement}

Consider a linear system

$$
\dot{x}=A x+B u, \quad y=C x,
$$

where $x \in \mathbb{R}^{n}, u \in \mathbb{R}^{m}, y \in \mathbb{R}^{l}, A, B, C$ are matrices of appropriate size. Let $\chi(\lambda)=C\left(I_{n}-A\right)^{-1} B$ be transfer matrix of the system (1) and $G$ be a prespecified $m \times l$-matrix.

Definition 1. System (1) is called G-passive if there exists a nonegative $n \times n$-matrix $P=P^{T} \geq 0$ such that

$$
V(x) \leq V\left(x_{0}\right)+\int_{0}^{t} u^{T}(t) G y(t) d t
$$

for any solution of system (1) satisfying $x(0)=x_{0}, x(t)=x$, where $V(x)=x^{T} P x$.

Definition 2. System (1) is called strictly G-passive, if there exist a positive definite $n \times n$-matrix $H=H^{T}>0$ and a positive number $\varrho>0$ such that

$$
V(x) \leq V\left(x_{0}\right)+\int_{0}^{t}\left[u(t)^{T} G y(t)-\varrho|x|^{2}\right] d t
$$


for any solution of system (1) satisfying $x(0)=x_{0}, x(t)=x$, where $V(x)=x^{T} H x$.

Obviously, if $l=m$ and $G=I_{m}$ is identity matrix, then $G$-passivity coincides with conventional passivity. This paper is concerned with studying only strict versions of $G$-passivity property.

Note that due to the smoothness of the system (1) and function $V(x)$, the integral inequalities (2), (3) are equivalent to their differential forms, e.g. (3) is equivalent to fullfillment of the differential dissipation inequality

$$
2 x^{T} H(A x+B u) \leq u^{T} G y-\varrho|x|^{2}
$$

for some $\varrho>0$ and all $x \in \mathbb{R}^{n}, u \in \mathbb{R}^{m}$. In its turn, the dissipation inequality (4) is, obviously, equivalent to the following matrix relations

$$
H A+A^{T} H<0, \quad H B=C^{T} G^{T} .
$$

The solvability conditions for 5 are given by the seminal Yakubovich-Kalman-Popov or Kalman-Yakubovich lemma (for this special case also called positive real lemma)

Consider the following passification problems.

Problem A. Find conditions of existence of $m \times l$-matrix $K$ and $m \times m$-matrix $L$ such that the system (1) with the output feedback

$$
u=K y+L v
$$

(where $v \in \mathbb{R}^{m}$ is new input, $\operatorname{det} L \neq 0$ ) is strictly $G$-passive.

Problem $B$. Find conditions of existence of $l \times m$-matrix $K$ such that the system (1) with the output feedback (6) is strictly $G$-passive with fixed matrix $L$.

Related problems for square MIMO systems were considered in $[11,12,14,13]$. In $[11,12]$ the special case $L=K$ was considered, while configuration in the papers $[14,13]$ corresponds to the case $L=I$.

In this paper the problems $\mathrm{A}$ and $\mathrm{B}$ for nonsquare systems $(l \neq m)$ are considered. Note that this case cannot be reduced to the "square" case by the "squaring down" procedure, see, e.g., [29], because intially the unknown matrix $K$ is rectangular, while squaring it down will reduce the number of constructive parameters. Remaining initial number of parameter is important, e.g. for adaptive control, where reducing the number of adjustable parameters may decrease transient performance of the adaptive systems, see $[21,23]$. It follows from the main results however (see next section) that squaring down by introducing new output $\bar{y}=G y \in \mathbb{R}^{m}$ does not change the passifiability conditions, while the passifying feedback can always be found in the form $u=\bar{K} \bar{y}+L v$.

\section{Main results}

In order to formulate the solutions to the above problems, introduce the following notations:

$$
\begin{gathered}
\delta(\lambda)=\operatorname{det}\left(\lambda I_{n}-A\right), \quad \chi(\lambda)=C^{*}\left(\lambda I_{n}-A\right)^{-1} B \\
\delta(\lambda, K)=\operatorname{det}\left[\lambda I_{n}-A(K)\right], \chi(\lambda, K)=C^{*}\left[\lambda I_{n}-A(K)\right]^{-1} B \\
\varphi(\lambda)=\delta(\lambda) \operatorname{det} G^{*} \chi(\lambda), \quad \Gamma=\lim _{\lambda \rightarrow \infty} \lambda G^{*} \chi(\lambda) .
\end{gathered}
$$

Obviously, $\delta(\lambda, K)$ and $\chi(\lambda, K)$ are characteristic polynomial and transfer matrix, respectively, of the system (1) closed with the feedback $u=K y+v$. It can be shown (see below Lemma 1) that $\varphi(\lambda)$ is a polynomial of degree $n-m$, invariant with respect to feedback transformation: substitution $\delta(\lambda, K)$ and $\chi(\lambda, K)$ instead of $\delta(\lambda)$ and $\chi(\lambda)$. Since $\Gamma=G^{*} C^{*} B$, the $m \times m$ matrix $\Gamma$ is also invariant with respect to the feedback transformation. Moreover, the following identities are valid:

$$
\begin{aligned}
& \delta(\lambda, K)=\delta(\lambda) \operatorname{det}\left[I_{m}-K^{*} \chi(\lambda)\right] \\
& \chi(\lambda, K)=\chi(\lambda)\left[I_{m}-K^{*} \chi(\lambda)\right]^{-1} .
\end{aligned}
$$

Definition 3. The system (1) is called minimum phase if the polynomial $\varphi(\lambda)$ is Hurwitz (its zeros belong to the open left half-plane). The (square) system is called strictly minimum phase if it is minimum phase and $\operatorname{det} C B \neq 0$, and hyper minimum phase if it is minimum phase and $C B=(C B)^{T}>0$.

Assume that rank $B=m$ which means just that there is no redundancy in inputs. The following two theorems give solutions to the Problems A, B.

Theorem 1. The system (1) is strictly G-passifiable by output feedback (6) if and only if it is minimum-phase and $\operatorname{det}(G C B) \neq 0$. (In other words, if and only if the transfer function $G \chi(\lambda)$ is strictly minimum-phase).

Theorem 2. The system (1) is strictly G-passifiable by output feedback (6) with fixed matrix $L$ if and only if it is minimumphase and $D=D^{T}>0$, where $D=G C B L$. (In other words, if and only if the transfer function $G \chi(\lambda) L$ is hyper-minimumphase).

Consider the following problem. Given complex-valued matrices $A, B, C, G, R$ of the dimensions $n \times n, n \times m, n \times l$, $l \times m$ and $n \times n$, respectively $(m \leq n, l \leq n)$. Additionally assume that $R=R^{*} \geq 0^{1}$. It is required to establish existence conditions for a Hermitian $n \times n$ matrix $H=H^{*}>0$ and a complex valued $l \times m$ matrix $K$ such that

$$
\begin{gathered}
H A(K)+A(K)^{*} H+R<0, \\
H B=C G
\end{gathered}
$$

where

$$
A(K)=A+B K^{*} C^{*} \text {. }
$$

The case when all the matrices $A, B, C, G, R$ are real valued will be called the real case.

\footnotetext{
${ }^{1} R^{*}$ means Hermitian-conjugate matrix, i.e., transposed matrix with complex conjugate elements; if $R$ is a real valued matrix, then $R^{*}=R^{T}$
} 
Proofs of the Theorems 1, 2 heavily rely on the following auxillary statement providing solvability conditions for corresponding matrix relations: inequality (9) and equalities (10), (11).

Theorem 3. For the existence of the matrices $H=H^{*}>0, K$ satisfying relations (9), (10), (11) and being real valued in the real case, it is sufficient (and, when $\operatorname{rank}(B)=m$, it is also necessary) that the matrix $G^{*} \chi(\lambda)$ be hyper minimum phase.

Obviously, relations (9), (10), (11) for fixed $K$ coincide with the linear matrix inequalities (LMI) (4) appearing in a version of Yakubovich-Kalman-Popov lemma. Therefore the Theorem 3 deals with the existence of a feedback rendering the system satisfy conditions of Yakubovich-Kalman-Popov lemma. In other words, the Theorem 3 can be called the Feedback Yakubovich-Kalman-Popov lemma. Note also that the inequalities (9) are bilinear matrix inequalities and the problem of their solvability is in general NP-hard. However for the above special case the solvability conditions for (9), (10), (11) are simple and constructive that is seen from the Theorem 3 formulation.

\section{Proof of main results}

Before proving the theorem, we formulate several auxiliary lemmas. Their proofs can be found in $[19,6]$

\section{Lemma 1. Let}

$$
\alpha(\lambda)=\left(\lambda I_{n}-A\right)^{-1} \delta(\lambda),
$$

$p, q$ be any $n \times m$ matrices and $\Sigma(\lambda)=p^{*} \alpha(\lambda) q$. Then $\operatorname{det} \Sigma(\lambda)=\delta^{m-1}(\lambda) \sigma(\lambda)$, where $\sigma(\lambda)$ is a polynomial of degree no higher then $n-m$ with the leading coefficient $\lambda^{n-m} \operatorname{det} p^{*} q$. Besides, $\sigma(\lambda)$ does not change with substitution $A+q r^{*}$ instead of $A$, where $r$ is any $n \times m$ matrix.

Corollary 1. The polynomial

$$
\varphi(\lambda)=\delta(\lambda) G^{*} \chi(\lambda)=G^{*} C^{*}\left[\left(\lambda I_{n}-A\right)^{-1} \delta(\lambda)\right] B,
$$

defined above, has the leading coefficient

$\lambda^{n-m} \operatorname{det} G^{*} C^{*} B$ and is invariant under "feedback transformation" of the form $A \rightarrow A(K)=A+B K^{*} C^{*}$.

Lemma 2. Any kth-order minor of the matrix $\Sigma(\lambda)$, defined in Lemma 1, is divisible by $\delta(\lambda)^{k-1}$, with the fraction being a polynomial of degree not exceeding $n-k$. Its leading coefficient is equal to the corresponding minor of the matrix $p^{*} q$.

Lemma 3. Let the polynomial $P_{\epsilon}(\lambda)$ have the form

$$
P_{\epsilon}(\lambda)=\lambda^{n-m} Q_{\epsilon}(\lambda)+R_{\epsilon}(\lambda),
$$

where

$Q_{\epsilon}(\lambda)=\sum_{k=0}^{m}\left(q_{k}+q_{k}^{\prime}(\epsilon)\right) \epsilon^{k} \lambda^{k}, R_{\epsilon}(\lambda)=\sum_{k=0}^{n-m-1}\left(r_{k}+r_{k}^{\prime}(\epsilon)\right) \lambda^{k}$, and $q_{k}^{\prime}(\epsilon)=O(\epsilon), r_{k}^{\prime}(\epsilon)=O(\epsilon)$ for $\epsilon \rightarrow 0$. Let, additionally, the polynomials

$$
Q(\lambda)=\sum_{k=0}^{m} q_{k} \lambda^{k}, \quad R(\lambda)=q_{0} \lambda^{n-m}+\sum_{k=0}^{n-m-1} r_{k} \lambda^{k}
$$

be Hurwitz. Then the polynomial $P_{\epsilon}(\lambda)$ is Hurwitz for all sufficiently small $\epsilon>0$.

Lemma 4. Let $\Phi(\omega), \omega \in \mathbb{R}^{1}$ be complex valued $m \times m$ matrix satisfying for any $x \neq 0 \omega \in \mathbb{R}^{1}$ the following inequality: $\operatorname{Re} x^{*} \Phi(\omega) x>0$. Then

$$
|\Delta \arg \operatorname{det} \Phi(\omega)| \leq m \pi,
$$

where

$$
\Delta \arg \Psi(\omega)=\lim _{\omega \rightarrow+\infty}[\arg \Psi(\omega)-\arg \Psi(-\omega)] .
$$

Finally, we will need a version of the Yakubovich-KalmanPopov lemma which can be easily derived from the results of [31].

Lemma 5. Let $A_{0}, R, B, Q$ be matrices of dimensions $n \times n$, $n \times n, n \times m, n \times m$ respectively, and $R=R^{*} \geq 0, \operatorname{rank} B=$ m. Let $^{2}$

$$
\begin{aligned}
& \Pi(\lambda)=2 \operatorname{Re} Q\left(\lambda I_{n}-A_{0}\right)^{-1} B- \\
& B^{*}\left(\lambda I_{n}-A_{0}\right)^{*-1} R\left(\lambda I_{n}-A_{0}\right)^{-1} B .
\end{aligned}
$$

For the existence of $n \times n$ matrix $H=H^{*}>0$ such that

$$
H A_{0}+A_{0} H+R<0, \quad H B=Q
$$

which is real valued in the real case, the following conditions are necessary and sufficient:

i) $\operatorname{det}\left(\lambda I_{n}-A_{0}\right)$ is a Hurwitz polynomial;

ii) $\Pi(i \omega)>0 \quad \forall \omega \in \mathbb{R}^{1}$,

iii) $\lim _{\omega \rightarrow \infty} \omega^{2} \Pi(i \omega)>0$.

Proof of Theorem 1. First, let us prove the sufficiency. To this end, choose an $l \times m$ matrix $K_{0}$ such that matrices $A_{0}=$ $A+B K_{0}^{*} C^{*}, Q=C G$ satisfy conditions i), ii), iii) of Lemma 5. In particular, $K_{0}$ can be chosen as $K_{0}=-k G$, where $k>0$ is sufficiently large (this guarantees that $K_{0}$ is real valued in the real case). For the sake of notational convenience we denote

$\delta_{k}(\lambda)=\delta(\lambda-k G), A_{k}=A-k B G^{*} C^{*}, \chi_{k}(\lambda)=\chi(\lambda-k G)$.

To check part ( $i$ ) we use the equality (7) which means that

$$
\delta_{k}(\lambda)=\frac{k^{m}}{\delta(\lambda)^{m-1}} \operatorname{det}\left[\frac{\delta(\lambda)}{k} I_{m}+G^{*} a(\lambda)\right],
$$

where $a(\lambda)=\chi(\lambda) \delta(\lambda)$. Expanding the determinant in the right hand part of expression(14) we obtain

$$
\begin{aligned}
& \delta_{k}(\lambda)=\frac{k^{m}}{\delta(\lambda)^{m-1}}\left[\frac{\delta(\lambda)^{m}}{k^{m}}+\varphi_{1}(\lambda) \frac{\delta(\lambda)^{m-1}}{k^{m-1}}+\cdots\right. \\
& \left.\quad+\varphi_{m-1}(\lambda) \frac{\delta(\lambda)}{k}+\varphi_{m}(\lambda)\right]
\end{aligned}
$$

where $\varphi_{1}(\lambda), \ldots, \varphi_{m-1}(\lambda), \varphi_{m}(\lambda)=\operatorname{det} G^{*} a(\lambda)$ are the coefficients of the characteristic polynomial of the matrix $G a(\lambda)$.

\footnotetext{
${ }^{2}$ Notation $\operatorname{Re} K$ stands for the Hermitian part of the matrix: $\operatorname{Re} K=(K+$ $\left.K^{*}\right) / 2$.
} 
By Lemma $2 \varphi_{k}(\lambda)=\delta(\lambda)^{k-1} \psi_{k}(\lambda)$, where $\psi_{k}(\lambda$ is a polynomial of degree $n-k$, the leading coefficient of which $\psi_{k}$ is equal to the sum of principal minors of order $k$ for the matrix $G^{*} C^{*} B(k=1, \ldots, m)$.

Therefore

$$
\begin{aligned}
& \frac{1}{k^{m}} \delta_{k}(\lambda)=\frac{\delta(\lambda)}{k^{m}}+\frac{\psi_{1}(\lambda)}{k^{m-1}}+\cdots+\frac{\psi_{m-1}(\lambda)}{k}+\psi_{m}(\lambda)= \\
& \lambda^{n-m}\left[\left(\frac{\lambda}{k}\right)^{m^{2}}+\left(\frac{\lambda}{k}\right)^{m-1}\left(\psi_{1}+O\left(\frac{1}{k}\right)\right)+\ldots\right. \\
& \left.+\left(\frac{\lambda}{k}\right)\left(\psi_{m-1}+O\left(\frac{1}{k}\right)\right)\right]+\psi_{m}(\lambda)+\psi(\lambda, k)
\end{aligned}
$$

where $\psi(\lambda, k)$ is some polynomial of degree not higher then $n-m$ with coefficients of order $O(1 / k)$ as $k \rightarrow \infty$. Applying Lemma 3 for $\epsilon=1 / k$, we obtain that the polynomial $\delta_{k}(\lambda)$ is Hurwitz for sufficiently large $k$, if the following polynomials are also Hurwitz

$$
Q(\lambda)=\lambda^{m}+\sum_{k=0}^{m-1} \psi_{k} \lambda^{k}, \quad R(\lambda)=\psi_{m}(\lambda) .
$$

However, $Q(\lambda)$ and $R(\lambda)$ are Hurwitz by assumptions of the theorem since

$$
Q(\lambda)=\operatorname{det}\left(\lambda I_{m}+G^{*} C^{*} B\right)=\operatorname{det}\left(\lambda I_{m}+\Gamma\right),
$$

while $R(\lambda)=\varphi(\lambda)$. Thus the part (i) is valid for $k>k_{1}$ and some positive number $k_{1}$.

To prove part (ii), let us rewrite it in the following form:

$$
2 \operatorname{Re} G^{*} \chi_{k}(i \omega)>B^{*}\left(-i \omega I_{n}-A_{k}^{*}\right)^{-1} R\left(i \omega I_{n}-A_{k}\right)^{-1} B .
$$

By virtue of invariance of the polynomial $\varphi(\lambda)$ for any $k$, we conclude that $\operatorname{det} G^{*} \chi_{k}(\lambda)=\varphi(\lambda) / \delta_{k}(\lambda)$. In accordance with the conditions of the theorem, the polynomial $\varphi(\lambda)$ is Hurwitz, and, hence,

$$
\operatorname{det} G^{*} \chi_{k}(i \omega) \neq 0 \quad \forall \omega \in \mathbb{R}^{1} .
$$

Therefore for any $\omega \in \mathbb{R}^{1}$ we obtain

$$
\left.\operatorname{Re} G^{*} \chi_{k}(i \omega)=G^{*} \chi_{k}(i \omega)\right]^{*} \operatorname{Re}\left[G^{*} \chi_{k}(i \omega)\right]^{-1} G^{*} \chi_{k}(i \omega) .
$$

In view of the latter equation, inequality (15) is equivalent to the following inequality

$$
\begin{aligned}
& 2 \operatorname{Re}\left[G^{*} \chi_{k}(i \omega)\right]^{-1}> \\
& {\left[\chi_{k}^{*}(i \omega) G\right]^{-1} B^{*}\left(-i \omega I_{n}-A_{k}\right)^{-1} B\left[G^{*} \chi_{k}(i \omega)\right]^{-1} .}
\end{aligned}
$$

However

$$
\left[G^{*} \chi_{k}(\lambda)\right]^{-1}=k I_{m}+\left[G^{*} \chi(\lambda)\right]^{-1} .
$$

Therefore it is sufficient to show that $\operatorname{Re}\left[G^{*} \chi(i \omega)\right]^{-1}$ and the right-hand side of (16) is bounded for $\omega \in \mathbb{R}^{1}$. Since $\operatorname{det} G^{*} \chi(i \omega)=\varphi(i \omega) / \delta(i \omega)$ for $\omega \in \mathbb{R}^{1}$, the matrix $\left[G^{*} \chi(i \omega)\right]^{-1}$ is bounded for any bounded change of $\omega$.

Now let us show that $\operatorname{Re}[G \chi(i \omega)]^{-1}$ is bounded for $\omega \rightarrow \pm \infty$. By virtue of the conditions of the theorem, $\Gamma=\Gamma^{*}>0$, where $\Gamma=G^{*} C^{*} B$. Therefore

$$
\begin{aligned}
& \operatorname{Re}\left[G^{*} \chi(i \omega)\right]^{-1}=\operatorname{Re} i \omega\left[i \omega G^{*} \chi(i \omega)\right]^{-1}=\operatorname{Re} i \omega\left[\Gamma^{-1}+\right. \\
& \left.O\left(\epsilon^{-1}\right)\right]=i \omega\left[\Gamma^{-1}+\left(\Gamma^{-1}\right)^{*}\right]+O(1)=O(1) \text { for } \omega \rightarrow \pm \infty
\end{aligned}
$$

To end the proof we need to show the boundedness of the righthand side of (16) for $\omega \rightarrow \pm \infty$ (for the notational convenience we denote the right hand side of (16) as $\psi_{k}(\omega)$ ). For $k>k_{1}$ the matrix function $\psi_{k}(\cdot)$ is continuous, and, hence, bounded on any bounded set. Let us show that for any $k$ there exists a finite limit $\lim _{\omega \rightarrow \pm \infty} \psi_{k}(\omega)$. Let $B_{\lambda}=\left(\lambda I_{n}-A_{k}\right)^{-1} B$. Then $B_{\lambda}=B / \lambda+O\left(1 /|\lambda|^{2}\right)$ for $\lambda \rightarrow \infty$, and, hence,

$$
\lim _{\omega \rightarrow \pm \infty} B_{i \omega}\left[G^{*} C^{*} B_{i \omega}\right]^{-1}=B\left[G^{*} C^{*} B\right]^{-1} .
$$

Therefore there exists a finite limit

$$
\begin{aligned}
\lim _{\omega \rightarrow \pm \infty} \psi_{k}(\omega) & =\lim _{\omega \rightarrow \pm \infty}\left[B_{i \omega}^{*} C G\right]^{-1} B_{i \omega}^{*} R B_{i \omega}\left[G^{*} C^{*} B_{i \omega}\right]^{-1} \\
& =\left[B^{*} C G\right]^{-1} B^{*} R B\left[G^{*} C^{*} B\right]^{-1}
\end{aligned}
$$

Thus, part (ii) of Lemma 5 is valid for $k>k_{2}$ and some positive number $k_{2}>k_{1}>0$.

Finally, the validity of part (iii) for sufficiently large $k>0$ follows from the following relationships which is easy to check

$$
\begin{aligned}
& \lim _{\omega \rightarrow \infty} \omega^{2} \Pi(i \omega)=-2 \operatorname{Re} G^{*} C^{*} A_{k} B-B^{*} R B \\
& =-2 \operatorname{Re} G^{*} C^{*} A B-B^{*} R B+2 k\left[G^{*} C^{*} B\right]^{2} .
\end{aligned}
$$

Thus the sufficiency part of the theorem is proved. Let us prove its necessity. Let relationships (9), (10), (11) be fulfilled for some $H_{0}=H_{0}^{*}>0$ and $K_{0}$. By Lemma 5 the polynomial $\delta_{0}(\lambda)=\operatorname{det}\left(\lambda I_{n}-A\left(K_{0}\right)\right)$ is Hurwitz and $\operatorname{Re} G^{*} \chi_{0}(i \omega)>0$ for any $\omega \in \mathbb{R}^{1}$, where $\chi_{0}(\lambda)=C^{*}\left[\lambda I_{n}-A\left(K_{0}\right)\right]^{-1} B$. Due to the invariance of the polynomial $\varphi(\lambda)$ we obtain

$$
\varphi(\lambda)=\delta_{0}(\lambda) \operatorname{det} G^{*} \chi_{0}(\lambda)
$$

Calculating the increment of the argument for $\lambda=i \omega$, where $\omega$ varies from $-\infty$ to $+\infty$ for both sides of this equality, we have

$$
\Delta \arg \varphi(i \omega)=n \pi+\Delta \arg \operatorname{det} G^{*} \chi_{0}(i \omega)
$$

By Lemma 4 we obtain $\Delta \arg \varphi(i \omega) \geq(n-m) \pi$. However $\varphi(\lambda)$ is a polynomial of degree $n-m$ with the leading coefficient $\lambda^{n-m} \operatorname{det} \Gamma$, where $\Gamma=G^{*} C^{*} B$ (see Lemma 1). Therefore $|\Delta \arg \varphi(i \omega)| \leq(n-m) \pi$, and $\Delta \arg \varphi(i \omega)=(n-m) \pi$. Hence $\varphi(\lambda)$ is Hurwitz, and $\operatorname{det} \Gamma>0$.

It remains to show that $\Gamma=\Gamma^{*} \geq 0$. By Lemma 5

$$
\begin{aligned}
& \lim _{\omega \rightarrow \infty} \omega^{2} \operatorname{Re} G^{*} \chi_{0}(i \omega)= \\
& \lim _{\omega \rightarrow \infty} \operatorname{Re}\left[i \omega G^{*} C^{*} B-G^{*} C^{*} A\left(K_{0}\right) B+O(1 /|\omega|)\right]= \\
& \lim _{\omega \rightarrow \infty} i \omega\left[\Gamma-\Gamma^{*}\right]-\operatorname{Re} G^{*} C^{*} A\left(K_{0}\right) B>0,
\end{aligned}
$$

and, hence, $\Gamma=\Gamma^{*}$. It is worth noting that the relationships (9), (10), (11) are, obviously, valid if we substitute $A\left(K_{0}\right)-$ $k I_{n},(k>0)$ instead of $A\left(K_{0}\right)$. Applying Lemma 5 again, we conclude that

$$
-G^{*} C^{*} A\left(K_{0}\right)+B+k \Gamma>0
$$


for any $k>0$. Therefore $\Gamma \geq 0$, which completes the proof of the theorem.

Proof of the Theorems 1, 2. Proof of the main results is now straightforward. If the matrix $L$ is fixed, then the strict output passifiability is equivalent to existence of matrices $K, H$, satisfying relations (9) - (11) where $B$ is replaced by $B L$. By Theorem 3 , it is equivalent to the hyper-minimum-phaseness of the transfer matrix $G \chi(\lambda) L$, i.e. to the minimum phaseness and fulfillment of $D=D^{T}>0$, where $D=G C B L$. If matrix $L$ is subject to choice, we can ensure the relation $D=D^{T}>0$ as soon as system is strictly minimum phase, i.e. if $\operatorname{det}(G C B) \neq 0$. In this case the simplest choice is $L=(G C B)^{-1}$.

Corollary 2. The set of matrices $K$ satisfying (9) - (11) i.e. passifying the initial system contains the subset of matrices of form $K=-\alpha G$, where

$$
\alpha \geq \alpha_{0}=\sup _{\omega \in \mathbb{R}^{1}} \lambda_{\min }[G W(j \omega)]^{-1}
$$

\section{Conclusions}

The paper recalls and clarifies some interrelations between passification of nonsquare linear systems and solvability of a class of bilinear matrix inequalities. The presented passification results can be useful for design purposes in various situations.

\section{References}

[1] Saberi A., Kokotovic P.V., Sussmann H.J. "Global stabilization of partially linear composite systems", SIAM J.Contr. Optimiz., 28, No 6, pp. 1491-1503, (1990).

[2] Byrnes C.I., Isidori A., Willems J.C. "Passivity, feedback equivalence, and the global stabilization of minimum phase nonlinear systems", IEEE Trans. Autom. Contr., 36, No. 11, pp. 1228-1240, (1991).

[3] Picci G., Pinzoni S. "On feedback dissipative systems", $J$. of Math. Systems and Control, 2, No. 1, pp. 1-30, (1992).

[4] Seron M.M., Hill D.J., Fradkov A.L. "Adaptive passification of nonlinear systems", In: Proc. 33rd Conf. on Decision and Control, pp.190-195, 1994.

[5] Sepulchre R., Janković M., Kokotović P.V. "Constructive Nonlinear Control”, New York, Springer-Verlag, (1996).

[6] Fradkov A.L., Miroshnik I.V., Nikiforov V.O. "Nonlinear and adaptive control of complex systems", Dordrecht: Kluwer Academic Publishers, (1999).

[7] Sobel K., Kaufman H.,Mabius L. "Implicit adaptive control for a class of MIMO systems", IEEE Trans. Aerosp. Electr. Syst., 18, pp. 576-590, (1982).

[8] Kaufman H., Bar-Kana I., Sobel K. "Direct Adaptive Control Algorithms". Springer, NY, 1994.
[9] Zeheb A. A sufficient condition for output feedback stabilization on uncertain systems, IEEE Trans Autom. Control, 31, No. 11, pp. 1055-1057.

[10] Steinberg A. "A sufficient condition for output feedback stabilization on uncertain dynamical systems", IEEE Trans Autom. Control, 33, No. 7, pp. 676-677, (1988).

[11] Gu G. "Stabilizability conditions of multivariable uncertain systems via output feedback control", IEEE Trans Autom. Control, 35, No. 8, pp. 925-927, (1990).

[12] Abdallah C., Dorato P., Karni S. "SPR design using feedback", In: Amer. Contr. Conf., pp.1742-1743, (1990).

[13] Huang C.-H., Ioannou P.I., Maroulas J., Safonov M.G. "Design of strictly positive real systems using constant output feedback", IEEE Trans Autom. Control, 44, No. 3, pp. 569-573, (1999).

[14] Weiss H., Wang Q., Speyer J.L. System characterization of positive real conditions. IEEE Trans Autom. Control, 39, No. 3, pp. 540-544, (1994).

[15] Kokotovic P.V., Sussmann H.J. "A positive real condition for global stabilization of nonlinear systems", Systems \& Control Letters, 13, pp. 125-133 (1989).

[16] Fradkov A.L., Hill D.J. "Exponential feedback passivity and stabilizability of nonlinear systems", Automatica, No.6. pp. 697-703, (1998).

[17] Sun W., Khargonekar P., Shim D. 'Solution to the positive real control problem for linear time-invariant systems", IEEE Trans Autom. Control, 39, No. 10, pp. 2034-2046, (1994).

[18] Fradkov, A.L. "Synthesis of an adaptive system for linear plant stabilization", Autom. Remote Control, 35, No. 12, pp. 1960-1966, (1974).

[19] Fradkov A.L. "Quadratic Lyapunov functions in the adaptive stabilization problem of a linear dynamic plant", Siberian Math. J., No. 2, pp. 341-348, (1976).

[20] Andrievsky B.R., Churilov A.N., Fradkov A.L. "Feedback Kalman-Yakubovich Lemma and its applications to adaptive control", Proc. 35th IEEE Conf. on Control and Decision, pp. 4537-4542, (1996).

[21] Fomin V.N., Fradkov A.L. and Yakubovich V.A. “ Adaptive Control of Dynamic Objects", Moscow: Nauka, (1981, in Russian).

[22] Tsykunov A.M. "Adaptive control of systems with delay", Moscow, Nauka, (1984 in Russian).

[23] Fradkov A.L. "Adaptive Control in Complex Systems". Moscow, Nauka, (1990, in Russian) 
[24] Andrievsky B.R., Stotsky A.A. Fradkov A.L. "Velocity gradient algorithms in control and adaptation problems. A survey", Autom. Rem. Control, No.12, pp. 1533-1563, (1988).

[25] Markov A.Yu., Fradkov A.L. "Adaptive synchronization of chaotic systems based on speed gradient method and passification", IEEE Trans. Circ. and Syst. 1997, No 10. pp. 905-912 (1997).

[26] Fradkov A.L., Pogromsky A.Yu., "Introduction to Control of Oscillations and Chaos", World Scientific, (1998).

[27] Fradkov A.L., Nijmeijer H., Markov A.Yu. "Adaptive observer-based synchronization for communication", Int. J. Bifurcations and Chaos, 10, No 12, (2000).

[28] Andrievsky, B.R., Fradkov A.L., Kaufman H. "Necessary and sufficient condition for almost strict positive realness and their application to direct implicit adaptive control". In: Proc. of Amer. Contr. Conf., Baltimore, pp. 12651266, (1994).

[29] Saberi A., Sannuti P. "Squaring down by static and dynamic compensators". IEEE Trans. Autom. Contr., 33, No 4, pp. 358-365 (1988).

[30] Popov V.M. "On the problem in the theory of absolute stability of automatic control", Autom. Remote Control, 25, No. 9, pp. 1129-1134, (1964).

[31] Yakubovich V.A. "The solution of certain matrix inequalities in automatic control theory", Soviet Mathematics. AMS, 3, pp. 620-623 (1962). 